\title{
The Feasibility of Recording Speech Breathing Patterns before and after Pulmonary Rehabilitation in an Out-Patient Environment
}

\section{Rokhsaneh Tehrany ${ }^{1, *}$, Anne Bruton ${ }^{1}$ and Anna Barney ${ }^{2}$}

${ }^{1}$ Faculty of Health Sciences, University of Southampton, Southampton, UK

${ }^{2}$ Institute of Sound and Vibration Research, University of Southampton, Southampton, UK

\begin{abstract}
This study aimed to determine the feasibility of recording speech breathing patterns before and after a six week Pulmonary Rehabilitation programme (PR) for patients with Chronic Obstructive Pulmonary Disease (COPD) and bronchiectasis. Fourteen patients with COPD (age $69 \pm 9.64$ ) and 6 patients with bronchiectasis (age $71 \pm 6.15$ ) were recruited from a respiratory outpatients department, and breathing patterns were recorded using Respiratory Inductive Plethysmography (RIP) during periods of quiet breathing, reading, counting and conversational speech, before and after a six week PR programme. Nine parameters were extracted from the recorded RIP signals: respiratory rate (bpm), inspiration and expiration time (s), inspiration and expiration magnitude (arbitrary units representing volume) and the regional percentage contributions of the ribcage and abdomen during inspiration and expiration. Clinical data, modified Borg scores, and Incremental Shuttle Walk Test (ISWT) data were retrieved from medical notes. Functional exercise capacity (ISWT) significantly improved following $P R$ by $89 \mathrm{~m}$ for the group $(t=-4.11, d f=16, p=0.00)$. No clinically or statistically significant changes in breathlessness (Borg), or speech breathing parameters, were detected following PR during any of the speech tasks. This study has demonstrated the feasibility of collecting speech breathing pattern data outside laboratory conditions. No firm conclusions can be drawn from the findings because of the uncontrolled nature of the study. It is therefore too soon to know if speech breathing patterns will be a useful tool for the remote monitoring of respiratory health in future.
\end{abstract}

Keywords: Speech; Speech breathing pattern; Breathing pattern; Respiratory inductive plethysmography; Pulmonary rehabilitation; Chronic obstructive pulmonary disease

\section{Introduction}

Breathing is often the first vital sign to alter in an acutely deteriorating patient, because changes in breathing pattern accompany many respiratory [1-3] and non-respiratory disorders [4]. Abnormal breathing patterns have also been observed in patients with chronic respiratory diseases such as Chronic Obstructive Pulmonary Disease (COPD) [5], bronchiectasis [6] and asthma [7]. Monitoring changes in individual breathing pattern should therefore provide useful information about changes in respiratory health.

Clinicians are aware that patients in respiratory distress often find it difficult or impossible to speak in complete sentences [8-11]. Patients become more breathless during speech because of the competition imposed by communicational needs and respiratory demand [12,13]. We hypothesised that objective recording of breathing pattern during speech activities (known as speech breathing patterns) could provide a useful non-invasive system for monitoring the progress of respiratory patients, or as an outcome measure for therapeutic interventions.

There is some limited evidence to suggest that speech breathing patterns differ between healthy individuals and patients with chronic respiratory disease $[12,13]$. In healthy adults, speech breathing patterns are typically characterised by having a short inspiratory phase (to minimise the silences), and prolonged expiratory phase (to extend the time available for speech). Patients with asthma, COPD and sarcoidosis seem to be unable to adapt their breathing patterns in favour of speech [12]. These patients produce significantly longer inspiratory phases (increasing the silences), shorter expiratory phases (reducing the time available for speech), faster respiratory rate and smaller tidal volumes. These findings suggest that speech breathing patterns alter as a result of pathology and may therefore be useful to monitor the progress of disease. If speech breathing patterns are to have any use as an outcome measure, however, there is a need to demonstrate their responsiveness to change. Pulmonary Rehabilitation (PR) was chosen as an appropriate intervention to test our hypothesis that speech breathing patterns would alter in response to an intervention designed to reduce breathlessness. Trials have shown that PR significantly improves exercise capacity, reduces breathlessness and hospital admissions, and improves health related quality of life, even though minimal effect on lung function is reported [14-16]. In addition, Binazzi et al. [8] reported that breathlessness during speech significantly reduced following a four week Pulmonary Rehabilitation (PR) programme. However, they used a subjective questionnaire to assess self-perceived breathlessness. There is a need, therefore, to assess speech breathing patterns objectively. Our research question was: Do objective measures of breathing and speech breathing patterns alter after a six week PR programme for patients with chronic respiratory disease? This was an uncontrolled exploratory study, in which the aim was to provide proof of principle data, and to assess the feasibility of the protocol, not to provide a definitive answer to the research question.

\section{Ethics}

Ethical approval was given by Southampton A Central Ethics Committee before the start of the study (Ethics number: 12/SC/0302). Written and signed consent was obtained from each patient on the first day of $\mathrm{PR}$.

\section{Methods}

\section{Design}

An observational, repeated measures design was used to obtain

*Corresponding author: Rokhsaneh Tehrany, Faculty of Health Sciences, University of Southampton, Highfield Campus, Building 45, Room 0001, Southampton, SO17 1BJ, UK, E-mail: r.tehrany@soton.ac.uk

Received December 15 , 2015; Accepted January 27, 2016; Published January 31 , 2016

Citation: Tehrany R, Bruton A, Barney A (2016) The Feasibility of Recording Speech Breathing Patterns before and after Pulmonary Rehabilitation in an OutPatient Environment. J Pulm Respir Med 6: 318. doi:10.4172/2161-105X.1000318

Copyright: $\odot 2016$ Tehrany R, et al. This is an open-access article distributed under the terms of the Creative Commons Attribution License, which permits unrestricted use, distribution, and reproduction in any medium, provided the original author and source are credited. 
breathing and speech breathing pattern data before and after a six week PR programme.

\section{Participants}

The aim was to recruit a convenience sample of 20 patients from a PR assessment clinic within a UK NHS hospital. Patients were eligible for inclusion in the study if they fulfilled the hospital criteria for PR and were enrolled onto a PR programme. Criteria for PR: decreased exercise tolerance; symptomatic, stable chronic lung disease; cardiovascularly stable (blood pressure no greater than 170/100); heart rate stable; no untreated arrhythmias; no new tachycardia); breathlessness of pulmonary origin; good motivation; medically stable; stable comorbidities.

\section{Pulmonary rehabilitation programme}

The PR programme in this study was typical of a standard outpatient programme in the UK. It was a cohort programme (a group of patients started and finished together) that lasted for a period of six weeks. Patients attended two (three hourly) supervised sessions per week. Exercise training was the cornerstone of the programme, with multidisciplinary educational sessions providing advice on nutrition, symptoms management and behavioural adaptations that were also scheduled each week. As part of the behavioural adaptation theme, one session of physiotherapy breathing retraining (lasting one hour) was provided, and consisted of a combination of pursed-lipped breathing, relaxation and diaphragmatic breathing exercises.

\section{Data collection}

Two sources of data were used: 1) some data were retrieved from the medical notes by the researcher (modified Borg Scores, ISWT test results, lung function tests, and physiological measurements) and 2) some data were obtained directly by the researcher (demographic data, breathing and speech breathing pattern recordings). The latter data were recorded on the first and last day of a six week PR programme using Respiratory Inductive Plethysmography (RIP). An Inductotrace system (Respitrace ${ }^{\oplus}$, Ambulatory Monitoring Inc. New York) was used to record respiratory parameters relating to timings, relative volumes and the regional contributions of the ribcage and abdomen during tidal breathing. These parameters were calculated from the cross sectional displacement of the ribcage and abdomen individually (regional contribution parameters) and from their sum (timing and volume parameters) during inspiration and expiration. After completing a basic questionnaire about age, sex and past medical history, participants were fitted with two elasticised belts (Inductobands) that were embedded with Teflon insulated wires. Each band was secured around the bare chest using a Velcro fastening at the level of the ribcage (below the axilla) and the abdomen (below the low the lowest vertebral rib). A custom-built analogue-to-digital converter was used to convert the signals acquired by the RIP to digital form. Calibration of respiratory parameters was performed offline using Qualitative Diagnostic Calibration (QDC) previously described by Sackner et al. [17].

\section{Recording procedure}

Once the RIP bands had been fitted, the cross sectional displacement of the ribcage and abdomen, individually and in sum, were recorded during two minute periods of: a) quiet breathing, b) reading, c) counting and d) conversational speech. The same task order was used for each participant and at each time point. Participants were informed that they could stop at any point during the recordings if they became breathless or uncomfortable. The RIP Inductobands were removed at the end of the last recording period. This procedure was repeated at the second time point (the last day of PR). Calibration of the recorded signals was performed offline using the Qualitative Diagnostic Calibration (QDC) procedure [17], prior to the extraction of the breathing parameters.

\section{Parameter extraction}

All breathing parameters were extracted using a customised peak detection algorithm written in Matlab (2009) ${ }^{\circ}$, by a postdoctoral research fellow from the Institute of Sound and Vibration Research, University of Southampton. Breathing parameters were calculated through the detection of the local minima and maxima (defined as the lowest and highest points respectively) of each individual displacement signal recorded from the RIP and from their sum. Eight breathing parameters were directly extracted from the recorded signals: inspiration and expiration time (TI and TE) in seconds (sec) defined as the time from a minimum to the next maximum and time from a maximum to the next minimum respectively, inspiration and expiration volume (IM and EM) expressed in arbitrary units and defined as the amplitude change from minimum to maximum and from maximum to minimum respectively, and the regional contributions of ribcage (\%RCinsp and \%RCexp) and abdomen (\%ABinsp and \%RCexp) defined as the amplitude change during inspiration or expiration in the $\mathrm{RC}$ or $\mathrm{AB}$ signal relative to the amplitude of the sum of the two, and expressed as a percentage. The mean of each parameter over the two minute recording was then calculated for each breathing parameter. A further parameter, respiratory rate (RR) in breaths per minute (bpm) was then calculated by summing the TI and TE for each cycle to get the total breathing cycle duration, and taking the reciprocal of the mean of this measure over the 2 minute recording period.

\section{Statistical analysis}

Descriptive statistics (mean and standard deviation) were calculated for each of the extracted breathing parameters to characterise the sample, however comparative analyses between the first and last day of PR were only performed on five breathing parameters (RR, TI, TE, EM, and \%RCExp. This is because a number of interdependences existed amongst the full set of parameters. For example, IM and EM are inevitably highly correlated, because the volume of air breathed in roughly equates to the volume of air breathed out. Reducing the number of statistical tests performed by analysing only the independent parameters reduces the probability of obtaining type I errors.

All breathing and speech breathing pattern data were found to be normally distributed when assessed using the Shapiro-Wilk tests and inspected visually using histograms. Paired comparisons using $t$ tests adjusted with Bonferroni corrections were performed with these data between the first and last day of PR. Clinical data were also compared between the first and last day of PR using Wilcoxon's rank test for related samples (modified Borg scores) and paired t tests (ISWT).

\section{Results}

Twenty patients were recruited, 14 had COPD (7 males) and six had bronchiectasis (1 male). Demographic and lung function data have been presented according to primary diagnosis (COPD or bronchiectasis) and from pooled results (referred to as 'patients with chronic respiratory disease (CRD)') (Table 1). On average, participants with COPD had their illness for longer (6.81 years) than those with bronchiectasis ( 4.40 years). All patients were considered to have 'severe' airflow obstruction based on the GOLD classification of airways obstruction guidelines $\left(\mathrm{FEV}_{1} \mathrm{pp}<50 \%\right)$ [18]. No statistically significant differences for any demographic or lung function data were identified between the two groups, when compared using independent $t$ tests. This finding supported the decision to pool the data from these two diagnostic groups for further analysis. 
Citation: Tehrany R, Bruton A, Barney A (2016) The Feasibility of Recording Speech Breathing Patterns before and after Pulmonary Rehabilitation in an Out-Patient Environment. J Pulm Respir Med 6: 318. doi:10.4172/2161-105X.1000318

\section{Self-perceived breathlessness (modified Borg Scores)}

Self-perceived breathlessness was rated on a 10-point modified Borg scale at rest by each participant on the first and last day of PR. Following a six week PR programme, the majority of patients reported the same level of breathlessness as at baseline (tie; $n=8$ ). Similar numbers of patients had a lower breathlessness score after PR (negative ranks; $n=5$ ), than before $P R$ (positive ranks $n=4$ ). Although the average modified Borg Score for the group was slightly higher after the six week PR programme, the actual difference was exceptionally small $(0.04)$ and was not statistically significant $(p=0.76)$. The average level of breathlessness reported by the group both before and after PR was very low in intensity (1="very mild shortness of breath").

\section{Functional walking distance (ISWT)}

Seventeen patients were included in the analysis comparing pre and post PR walking distance during the ISWT. Three patients did not participate in both tests (one missed the before PR test and two missed the after PR test), but the reasons for this were not documented in the medical notes. On average, the whole group increased their walking distance by $88.82 \mathrm{~m}$ following a six week PR programme. This finding was found to be statistically significant $(\mathrm{p}<0.0005)$ (Table 2$)$.

\section{Breathing and speech breathing pattern data}

Breathing and speech breathing parameters were characterised during each two minute task on the first and last day of a six week PR programme (Tables 3-6). Paired t tests adjusted with Bonferroni corrections were performed between the first and last day of $\mathrm{PR}$, and a result was considered as statistically significant if $\mathrm{p}<0.01$.

No statistically significant differences were found between the first and last PR session ( $p>0.01)$ for any of the tasks or parameters examined. Individual data were then examined. The direction of change was not found to be consistent for any of the breathing parameters and tasks. Therefore, these findings indicate that breathing and speech breathing patterns did not change in either a statistically or clinically significant manner after PR.

\begin{tabular}{|c|c|c|c|}
\hline & COPD $(\mathbf{n = 1 4 )}$ & $\begin{array}{c}\text { Bronchiectasis } \\
(\mathbf{n}=6)\end{array}$ & $\begin{array}{c}\text { Pooled patients } \\
\text { with CRD }(\mathbf{n}=\mathbf{2 0})\end{array}$ \\
\hline $\begin{array}{c}\text { Age (years) Mean } \\
\pm \text { SD }\end{array}$ & $69.36 \pm 9.64$ & $70.50 \pm 7.23$ & $69.70 \pm 8.81$ \\
\hline Sex & $M=7, F=7$ & $M=1, F=5$ & $M=8, F=12$ \\
\hline $\begin{array}{c}\text { Length of illness } \\
\text { (years) Mean Range }\end{array}$ & $6.81(1-14)$ & $4.40(1-10)$ & $6.14(1-14)$ \\
\hline $\begin{array}{c}\text { FEV } 1 \% \text { of predicted) } \\
\text { Mean } \pm \text { SD }\end{array}$ & $\begin{array}{c}46.43 \pm 14.82 \\
(n=7)\end{array}$ & $\begin{array}{c}51.56 \pm 10.17 \\
(n=4)\end{array}$ & $\begin{array}{c}48.36 \pm 13.04 \\
(n=11)\end{array}$ \\
\hline $\begin{array}{c}\text { FVC }(\% \text { of predicted) } \\
\text { Mean } \pm \text { SD }\end{array}$ & $\begin{array}{c}72.00 \pm 21.97 \\
(n=7)\end{array}$ & $\begin{array}{c}81.50 \pm 10.17 \\
(n=4)\end{array}$ & $\begin{array}{c}75.45 \pm 18.59 \\
(n=11)\end{array}$ \\
\hline FEV $/$ FVC & $\begin{array}{c}0.53 \pm 0.16 \\
(n=7)\end{array}$ & $0.48 \pm 0.11(n=4)$ & $0.51 \pm 0.14(n=11)$ \\
\hline
\end{tabular}

Table 1: Demographic and lung function data according to primary diagnosis and pooled results $n=20$

\begin{tabular}{|c|c|c|c|c|c|c|c|}
\hline & $\begin{array}{c}\text { Before } \\
\text { PR }\end{array}$ & After PR & $\begin{array}{c}\text { Mean } \\
\text { difference }\end{array}$ & $\begin{array}{c}95 \% \text { CI } \\
\text { Upper; } \\
\text { Lower }\end{array}$ & t & df & $\boldsymbol{p}$ \\
\hline $\begin{array}{c}\text { Walking } \\
\text { distance } \\
\text { (M) }\end{array}$ & $\begin{array}{c}166.67 \pm \\
88.73\end{array}$ & $\begin{array}{c}255.29 \pm \\
135.46\end{array}$ & 88.82 & $\begin{array}{c}-132.82 ; \\
-44.82\end{array}$ \\
SD
\end{tabular}

Table 2: Mean distance travelled during the ISWT before and after Pulmonary Rehabilitation in patients with COPD and bronchiectasis $n=17$.

\begin{tabular}{|c|c|c|c|c|c|c|c|}
\hline & Before PR & After PR & $\begin{array}{c}\text { Mean } \\
\text { difference }\end{array}$ & $\begin{array}{c}95 \% \mathrm{Cl} \\
\text { Upper; } \\
\text { lower }\end{array}$ & $\mathbf{t}$ & $\mathbf{d f}$ & $p$ \\
\hline $\mathrm{T}_{\mathbf{1}}(\mathrm{sec})$ & $1.56 \pm 0.58$ & $1.50 \pm 0.34$ & 0.06 & $-0.26 ; 0.39$ & 0.41 & 13 & 0.68 \\
\hline $\mathrm{T}_{\mathrm{E}}(\mathrm{sec})$ & $2.31 \pm 0.79$ & $2.17 \pm 0.54$ & 0.14 & $-0.21 ; 0.49$ & 0.86 & 13 & 0.40 \\
\hline IM (a.u) & $1.29 \pm 0.50$ & $1.52 \pm 0.62$ & - & - & - & - & \\
\hline EM (a.u) & $1.29 \pm 0.51$ & $1.50 \pm 0.60$ & -0.20 & $-0.57 ; 0.16$ & 1.21 & 13 & 0.24 \\
\hline RR (bpm) & $16.78 \pm 4.54$ & $17.11 \pm 3.99$ & -0.32 & $-2.48 ; 1.83$ & -0.32 & 13 & 0.75 \\
\hline \%RCInsp & $63.02 \pm 12.84$ & $66.56 \pm 13.21$ & - & - & - & - & - \\
\hline \%ABInsp & $36.29 \pm 13.05$ & $32.92 \pm 13.27$ & - & - & - & - & - \\
\hline \%RCExp & $61.51 \pm 13.37$ & $65.68 \pm 12.40$ & -4.61 & $-13.27 ;-$ & -2.28 & 13 & 0.04 \\
\hline \%ABExp & $37.81 \pm 13.90$ & $33.83 \pm 12.49$ & - & - & - & - & - \\
\hline
\end{tabular}

Table 3: Breathing parameters during a two minute quiet breathing task in patients with chronic respiratory disease. Descriptive statistics and results from the paired $t$ tests $(n=14)$

Descriptive statistics are provided for all nine breathing parameters. Comparative analyse s have only been performed on five selected parameters, within the highlighted rows in this table. $T_{1}(\mathrm{sec})=$ Inspiration time (seconds); $T_{E}(\mathrm{sec})=$ expiration time (sec); $\mathrm{IM}(\mathrm{a} . \mathrm{u})=$ inspiration magnitude (arbitrary units); $\mathrm{EM}(\mathrm{a} . \mathrm{u})=$ Expiration magnitude (arbitrary units); Ttot (sec)=breathing cycle time $(\mathrm{sec}) ; \mathrm{RR}(\mathrm{bpm})=$ respiratory rate (breaths per minute); \%RCInsp=Regional contribution of the ribcage to inspiration $\% A B I n s p=$ Regional contribution of the abdomen to inspiration; \%RCExp=Regional contribution of the ribcage to expiration; \%ABExp=Regional contribution of the abdomen to expiration (Table 3)

\begin{tabular}{|c|c|c|c|c|c|c|c|}
\hline & $\begin{array}{c}\text { Before PR } \\
\quad(n=18)\end{array}$ & $\begin{array}{c}\text { After PR } \\
(n=18)\end{array}$ & $\begin{array}{c}\text { Mean } \\
\text { difference }\end{array}$ & $\begin{array}{l}95 \% \mathrm{Cl} \\
\text { Upper } \\
\text {;lower }\end{array}$ & $\mathbf{t}$ & df & $p$ \\
\hline$T_{1}(\sec )$ & $0.65 \pm 0.13$ & $0.65 \pm 0.14$ & -0.005 & $-0.09 ; 0.08$ & -0.12 & 17 & 0.90 \\
\hline$T_{E}(s e c)$ & $3.22 \pm 0.71$ & $3.12 \pm 0.62$ & 0.10 & $-0.21 ; 0.42$ & 0.68 & 17 & 0.50 \\
\hline IM (a.u) & $1.34 \pm 0.38$ & $1.42 \pm 0.63$ & - & - & - & - & - \\
\hline EM (a.u) & $1.33 \pm 0.38$ & $1.40 \pm 0.62$ & -0.07 & $-0.42 ; 0.28$ & -0.41 & 17 & 0.68 \\
\hline RR (bpm) & $16.00 \pm 2.91$ & $16.40 \pm 3.04$ & -0.40 & $-1.91 ; 1.11$ & -0.55 & 17 & 0.58 \\
\hline \%RCInsp & $63.71 \pm 11.57$ & $65.49 \pm 12.96$ & - & - & - & - & - \\
\hline$\%$ ABInsp & $36.08 \pm 11.57$ & $34.29 \pm 12.94$ & - & - & - & - & - \\
\hline$\%$ RCExp & $63.06 \pm 15.50$ & $65.41 \pm 14.64$ & -2.34 & $-11.36 ; 6.67$ & -0.54 & 17 & 0.59 \\
\hline$\%$ ABExp & $36.81 \pm 15.37$ & $34.57 \pm 15.13$ & - & - & - & - & \\
\hline
\end{tabular}

Table 4: Breathing parameters during a two minute reading task in patients with chronic respiratory disease. Descriptive statistics and results from the paired $t$ tests $(n=18)$.

Descriptive statistics are provided for all nine breathing parameters. Comparative analyses have onlybeen performed on five selected parameters, within the highlighted rows in this table $T_{1}(\mathrm{sec})=$ Inspiration time (seconds); $T_{E}(\mathrm{sec})=$ expiration time (sec) $\mathrm{IM}(\mathrm{a} . \mathrm{u})=$ inspiration magnitude (arbitrary units); $\operatorname{EM}(\mathrm{a} . \mathrm{u})=$ Expiration magnitude (arbitrary units); Ttot (sec)=breathing cycle time (sec); RR(bpm)=respiratory rate (breaths per minute); \%RCInsp=Regional contribution of the ribcage to inspiration $\% A B I n s p=$ Regional contribution of the abdomen to inspiration; \%RCExp=Regional contribution of the ribcage to expiration; \%ABExp=Regional contribution of the abdomen to expiration (Table 4)

\section{Discussion}

This was the first study to examine speech breathing patterns before and after an intervention, in this case a PR programme for patients with chronic respiratory disease. Speech breathing patterns may be related to pulmonary function because there is some limited evidence to suggest that these patterns significantly differ between healthy individuals and patients with chronic respiratory disease $[12,13]$. We have established that the protocol is feasible within this patient group and that good data can be obtained outside the laboratory environment. The main research finding was that speech breathing patterns did not alter after a six week PR programme for patients with either COPD or bronchiectasis in this sample. This finding was consistent for every breathing parameter during each task. In addition, no changes in resting breathlessness (modified Borg scores) were detected after PR, 
whereas functional walking distance increased (both clinically and statistically significantly) after PR. This latter finding is in line with previous findings supporting the efficacy of PR [14,19,20]. Walking distance assessed via the ISWT increased by an average of $89 \mathrm{~m}$. This change exceeded the Minimal Clinically Important Difference (MCID) previously reported for the ISWT [21], where a change of $47.5 \mathrm{~m} \mathrm{(5}$ shuttles) was associated with feeling 'slightly' better, and a change of $78.7 \mathrm{~m}$ (eight shuttles) was associated with the next rating, "better". Although lung function was not measured following PR, the improved exercise capacity provides some evidence for the effectiveness of the PR programme involved in our study. Lung function tests are not considered to be a useful outcome measure following PR, as RCTs have repeatedly shown that improvements in patient centred outcomes are independent of changes in lung function [14-16]. However, despite the improvement in walking distance, in our study modified Borg scores did not significantly change between the first and last PR assessment. Most studies that have observed significant improvements in modified Borg Scores following PR have reported higher breathlessness scores at baseline (>5) [14,22-24]. In contrast, the average resting modified Borg Score in this study was found to be very low at baseline (equating to 'very mild shortness of breath'), and remained low after the six week PR programme. It is therefore likely that significant changes in breathlessness were not seen because the average modified Borg Score was already so low at baseline that there was no room for further improvement.

Breathlessness is related to specific parameters of breathing pattern, such as respiratory rate, timing components and lung volume $[25,26]$. The lack of detectable change between baseline and post PR speech breathing parameters could therefore also have been because there was no room for improvement. Although no studies have previously quantified speech breathing patterns before and after a PR programme, there is some limited evidence to suggest that speaking related breathlessness reduces following PR [8]. Binazzi et al. [8] evaluated the level of self-perceived breathlessness during speech in 31 patients with mild to severe COPD, before and after PR. Breathlessness during speech was evaluated using a 10 point questionnaire previously proposed by Lee et al. [11]. Each questionnaire item depicted a situation related to speech production, such as 'having a conversation with someone' and 'talking on the telephone', where patients were asked to rate each item based on their perception of breathlessness.

\begin{tabular}{|c|c|c|c|c|c|c|c|}
\hline & $\begin{array}{c}\text { Before PR } \\
(n=18)\end{array}$ & $\begin{array}{c}\begin{array}{c}\text { After PR } \\
(\mathrm{n}=18)\end{array}\end{array}$ & $\begin{array}{c}\text { Mean } \\
\text { difference }\end{array}$ & $\begin{array}{l}95 \% \mathrm{Cl} \\
\text { Upper } \\
\text {;lower }\end{array}$ & $t$ & df & $p$ \\
\hline$T_{1}(\mathrm{sec})$ & $0.72 \pm 0.18$ & $0.72 \pm 0.15$ & 0.005 & $-0.08 ; 0.09$ & 0.11 & 17 & 0.90 \\
\hline$T_{E}(\sec )$ & $3.74 \pm 1.09$ & $3.86 \pm 1.25$ & -0.11 & $-0.58 ; 0.34$ & -0.54 & 17 & $0.5 \mathrm{c}$ \\
\hline IM (a.u) & $1.46 \pm 0.61$ & $1.70 \pm 0.61$ & - & - & - & - & - \\
\hline EM (a.u) & $1.44 \pm 0.60$ & $1.67 \pm 0.59$ & -0.23 & $-0.57 ; 0.11$ & -1.40 & 17 & 0.18 \\
\hline RR (bpm) & $14.21 \pm 3.22$ & $13.95 \pm 3.44$ & 0.26 & $-0.76 ; 1.28$ & 0.53 & 17 & 0.59 \\
\hline \%RCInsp & $59.41 \pm 13.78$ & $61.32 \pm 8.09$ & - & - & - & - & - \\
\hline \%ABInsp & $40.40 \pm 13.67$ & $38.64 \pm 7.84$ & - & - & - & - & - \\
\hline \%RCExp & $58.50 \pm 13.89$ & $57.84 \pm 16.53$ & 0.65 & $-12.46 ; 5.18$ & -0.88 & 17 & 0.39 \\
\hline \%ABExp & $41.42 \pm 13.60$ & $41.77 \pm 16.19$ & - & - & - & - & - \\
\hline
\end{tabular}

Table 5: Breathing parameters during a two minute conversational speech task in patients with chronic respiratory disease. Descriptive statistics and results from the paired $t$ tests $(n=18)$.

Descriptive statistics are provided for all nine breathing parameters. Comparative analyses have only been performed on five selected parameters, within the highlighted rows in this table $T_{1}(\mathrm{sec})=$ Inspiration time (seconds); $T_{E}(\mathrm{sec})=$ expiration time (sec); IM(a.u)=inspiration magnitude (arbitrary units); $E M(a . u)=E x p i r a t i o n$ magnitude (arbitrary units); Ttot(sec)=breathing cycle time (sec); RR(bpm)=respiratory rate (breaths per minute); \%RCInsp=Regional contribution of the ribcage to inspiration $\% A B I n s p=$ Regional contribution of the abdomen to inspiration; \%RCExp=Regiona contribution of the ribcage to expiration; \%ABExp=Regional contribution of the abdomen to expiration (Table 5)

\begin{tabular}{|c|c|c|c|c|c|c|c|}
\hline & $\begin{array}{c}\text { Before PR } \\
(n=18)\end{array}$ & $\begin{array}{c}\text { After PR } \\
(\mathrm{n}=18)\end{array}$ & $\begin{array}{c}\text { Mean } \\
\text { difference }\end{array}$ & $\begin{array}{c}95 \% \mathrm{Cl} \\
\text { Upper } \\
\text {;lower }\end{array}$ & $\mathbf{t}$ & $\mathbf{d f}$ & $p$ \\
\hline $\mathbf{T}_{\mathbf{1}}$ (sec) & $0.64 \pm 0.25$ & $0.58 \pm 0.14$ & 0.06 & $-0.04 ; 0.17$ & 1.25 & 17 & 0.22 \\
\hline $\mathbf{T}_{\mathbf{E}}$ (sec) & $3.55 \pm 1.82$ & $3.66 \pm 1.47$ & -0.11 & $-0.77 ; 0.54$ & -0.36 & 17 & 0.71 \\
\hline IM (a.u) & $1.21 \pm 0.53$ & $1.30 \pm 0.72$ & - & - & - & - & - \\
\hline EM (a.u) & $1.21 \pm 0.52$ & $1.30 \pm 0.73$ & -0.09 & $-0.50 ; 0.31$ & -0.47 & 17 & 0.64 \\
\hline RR (bpm) & $16.37 \pm 5.54$ & $15.91 \pm 5.70$ & 0.46 & $-2.10 ; 3.03$ & 0.38 & 17 & 0.70 \\
\hline \%RCInsp & $56.96 \pm 13.63$ & $65.00 \pm 13.40$ & - & - & - & - & - \\
\hline \%ABInsp & $40.65 \pm 11.81$ & $37.52 \pm 12.40$ & - & - & - & - & - \\
\hline \%RCExp & $59.03 \pm 11.78$ & $62.22 \pm 12.60$ & -3.19 & $-11.79 ; 5.40$ & -0.78 & 17 & 0.44 \\
\hline \%ABExp & $40.65 \pm 11.81$ & $37.52 \pm 12.40$ & - & - & - & - & - \\
\hline
\end{tabular}

Table 6: Breathing parameters during a two minute counting task in patients with chronic respiratory disease. Descriptive statistics and results from the paired tests $(n=18)$.

Descriptive statistics are provided for all nine breathing parameters. Comparative analyses have only been performed on five selected parameters, within the highlighted rows in this table $T_{1}(\mathrm{sec})=$ Inspiration time (seconds); $T E(\mathrm{sec})=$ expiration time (sec); $\operatorname{IM}(\mathrm{a} . \mathrm{u})=$ =inspiration magnitude (arbitrary units); EM (a.u)=Expiration magnitude (arbitrary units); Ttot $(\mathrm{sec})=$ breathing cycle time $(\mathrm{sec}) ; \mathrm{RR}(\mathrm{bpm})=$ respiratory rate (breaths per minute); \%RCInsp=Regional contribution of the ribcage to inspiration; $\% A B I n s p=$ Regional contribution of the abdomen to inspiration; \%RCExp=Regional contribution of the ribcage to expiration; \%ABExp=Regional contribution of the abdomen to expiration (Table 6)

Although the reported level of breathlessness during speech was found to reduce significantly following the intervention (\% max before $\mathrm{PR}=60.3 \pm$ 23.2 compared after $\mathrm{PR}=43.7 \pm 19.7, \mathrm{p}<0.0001$ ), these findings may have been influenced by the high intensity of the PR programme in their study, as patients attended six sessions per week for four weeks. In contrast, the PR programme incorporated in our study involved two sessions per week, for a period of six weeks. Therefore, while we did not find any evidence to suggest that speech breathing patterns are responsive to a PR programme, it is possible that significant changes in breathlessness and speech breathing parameters were not observed because the intensity and duration of the PR programme was insufficient to generate change. A further limitation of our study relates to the small sample size, which meant there was insufficient statistical power to detect a genuine change over time in any of the parameters. Retrospective power calculations using our data result in different sample sizes depending on the breathing pattern parameter of interest. For example, calculations based on abdominal contributions to volume indicate that a sample size of 127 participants completing the programme would be required to detect a difference of $5 \%$ contribution at $80 \%$ power during a conversational speech task. Calculations based on expiratory time indicate a sample size of 30 completing the programme would be sufficient to detect a difference of 0.5 seconds at $80 \%$ power during a conversational speech task.

\section{Future Research}

It is acknowledged that a longer follow up period may be needed to detect changes after any intervention. Future studies should therefore aim to follow patients for up to one year after the intervention period. It would also be useful to include patients with different chronic respiratory pathologies to establish if different pathologies have different responses to the intervention.

\section{Conclusion}

The analysis of speech breathing patterns has theoretical potential as a respiratory monitoring tool, and/or as an outcome measure for evaluating respiratory health following a therapeutic intervention. This is the first study to have quantified speech breathing patterns before and after a PR programme for patients with chronic respiratory disease, to examine their responsiveness to change following the intervention. Although functional 
Citation: Tehrany R, Bruton A, Barney A (2016) The Feasibility of Recording Speech Breathing Patterns before and after Pulmonary Rehabilitation in an Out-Patient Environment. J Pulm Respir Med 6: 318. doi:10.4172/2161-105X.1000318

exercise capacity was found to improve significantly following PR, no changes were observed for breathlessness or speech breathing parameters. We have established that the protocol is feasible within this patient group and that good data can be obtained outside the laboratory environment. No firm conclusions can be drawn from the results because of the uncontrolled nature of the study, and because this small sample may not be representative of the wider PR population. It is therefore too soon to know if speech breathing patterns will be a useful tool for the remote monitoring of respiratory health in future. Clinicians know that patients' ability to form full sentences becomes impaired when they are breathless, but it is not yet known how speech breathing patterns relate to any underlying pathology, or how they respond to specific interventions. Larger, controlled studies are needed to examine this possibility.

\section{Acknowledgements}

The authors would like to thank Dr. Nikolic for her invaluable input during the data extraction phase, Dr. Ewings, for all the statistical support he provided, the respiratory therapists at St Richard's Hospital NHS Foundation Trust for their role during the recruitment phase, and all the participants for all their time and patience while taking part in the study.

\section{References}

1. Ashutosh K, Gilbert R, Auchincloss JH Jr, Peppi D (1975) Asynchronous breathing movements in patients with chronic obstructive pulmonary disease. Chest 67: 553-557.

2. Gilmartin JJ, Gibson GJ (1984) Abnormalities of chest wall motion in patients with chronic airflow obstruction. Thorax 39: 264-271.

3. Renzi G, Milic-Emili J, Grassino AE (1986) Breathing pattern in sarcoidosis and idiopathic pulmonary fibrosis. Ann N Y Acad Sci 465: 482-490.

4. Lieber C, Mohsenin $V(1992)$ Cheyne-Stokes respiration in congestive hear failure. Yale J Biol Med 65: 39-50.

5. Loveridge $B$, West $P$, Kryger $M H$, Anthonisen NR (1986) Alteration in breathing pattern with progression of chronic obstructive pulmonary disease. Am Rev Respir Dis 134: 930-934.

6. Koulouris NG, Retsou S, Kosmas E, Dimakou K, Malagari K, et al. (2003) Tidal expiratory flow limitation, dyspnoea and exercise capacity in patients with bilateral bronchiectasis. Eur Respir J 21: 743-748.

7. Ritz T, Simon E, Trueba AF (2011) Stress-induced respiratory pattern changes in asthma. Psychosom Med 73: 514-521.

8. Binazzi B, Lanini B, Romagnoli I, Garuglieri S, Stendardi L, et al. (2011) Dyspnoea during speech in Chronic Obstructive Pulmonary Disease patients: Effects of pulmonary rehabilitation. Respiration 81: 379-385.

9. Mahler DA, Wells CK (1988) Evaluation of clinical methods for rating dyspnea Chest 93: 580-586.
10. Kennedy S (2007) Detecting changes in the respiratory status of ward patients. Nurs Stand 21: 42-46.

11. Lee L, Friesen M, Lambert IR, Loudon RG (1998) Evaluation of dyspnea during physical and speech activities in patients with pulmonary diseases. Chest 113 625-632.

12. Lee L, Loudon RG, Jacobson BH, Stuebing R (1993) Speech breathing in patients with lung disease. Am Rev Respir Dis 147: 1199-1206.

13. Loudon RG, Lee L, Holcomb BJ (1988) Volumes and breathing patterns during speech in healthy and asthmatic subjects. J Speech Hear Res 31: 219-227.

14. Lacasse Y, Martin S, Lasserson TJ, Goldstein RS (2007) Meta-analysis of respiratory rehabilitation in chronic obstructive pulmonary disease. A Cochrane systematic review. Europa medicophysica 43: 475-485.

15. Wedzicha J, Bestall J, Garrod R, Garnham R, Paul E, et al. (1998) Randomized controlled trial of pulmonary rehabilitation in severe chronic obstructive pulmonary disease patients, stratified with the MRC dyspnoea scale. Eur Respir J 12: 363-369.

16. Sewell L, Singh SJ, Williams JE, Collier R, Morgan MD (2006) How long should outpatient pulmonary rehabilitation be? A randomised controlled trial of 4 weeks versus 7 weeks. Thorax 61: 767-771.

17. Sackner MA, Watson $H$, Belsito AS, Feinerman D, Suarez M, et al. (1989) Calibration of respiratory inductive plethysmograph during natural breathing. J Appl Phys 66: 410-420.

18. Decramer M (2014) Global strategy for the diagnosis, management and prevention of Chronic Obstructive Pulmonary Disease. Global Initiave fo Chonic Obstructive Lung Disease (GOLD).

19. Bush A, Pavord I (2013) British Thoracic Society guideline on Pulmonary Rehabilitation in adults. Thorax 68: 1-36.

20. Spruit MA, Singh SJ, Garvey C, ZuWallack R, Nici L, et al. (2013) An official American Thoracic Society/European Respiratory Society statement: key concepts and advances in pulmonary rehabilitation. Am J Respir Crit Care Med 188: e13-64.

21. Singh SJ, Jones PW, Evans R, Morgan MD (2008) Minimum clinically important improvement for the incremental shuttle walking test. Thorax 63: 775-777.

22. Ries AL (2005) Minimally clinically important difference for the UCSD Shortness of Breath Questionnaire, Borg Scale, and Visual Analog Scale. COPD 2: 105 110

23. Clini EM, Crisafulli E, Costi S, Rossi G, Lorenzi C, et al. (2009) Effects of early inpatient rehabilitation after acute exacerbation of COPD. Respir Med 103 : 1526-1531.

24. Foglio K, Bianchi L, Bruletti G, Battista L, Pagani M, et al. (1999) Long-term effectiveness of pulmonary rehabilitation in patients with chronic airway obstruction. Eur Respir J 13: 125-132.

25. Muers M (1993) Understanding breathlessness. Lancet 342: 1190-1191.

26. Rao AB, Gray D (2003) Breathlessness in hospitalised adult patients. Postgrad Med J 79: 681-685. 